\title{
The dynamic changes in the pattern of liver function tests in pregnant obese women
}

\author{
CRISTINA OANA DACIANA TEODORESCU ${ }^{1}$, FLORICA ȘANDRU ${ }^{2,3}$, ADHAM CHARKAOUI $^{4}$, \\ ANDREI TEODORESCU ${ }^{5}$, AMORIN REMUS POPA ${ }^{6}$ and ANDREEA-IULIANA MIRON ${ }^{2,7}$
}

\begin{abstract}
${ }^{1}$ Department of Obstetrics and Gynecology, 'Polizu' Clinical Hospital, 011061 Bucharest; ${ }^{2}$ Department of Dermatology, 'Carol Davila' University of Medicine and Pharmacy, 050474 Bucharest; ${ }^{3}$ Department of Dermatology, 'Elias' Emergency University Hospital, 011461 Bucharest; ${ }^{4}$ Department of Morphological and Functional Sciences, Faculty of Medicine and Pharmacy, 'Dunarea de Jos' University, 800008 Galati; ${ }^{5}$ Department of Morphology, University of Medicine and Pharmacy, 410028 Oradea; ${ }^{6}$ Department of Diabetes, Nutrition, Metabolic and Internal Diseases, University of Medicine and Pharmacy, 410033 Oradea; ${ }^{7}$ Department of Radiology, Oncology, Hematology, 'Colțea' Clinical Hospital, 927180 Bucharest, Romania
\end{abstract}

Received April 9, 2021; Accepted May 10, 2021

DOI: $10.3892 / \mathrm{etm} .2021 .10418$

\begin{abstract}
Obesity is an important problem in healthcare regarding gestating women. The objective of the present study was to highlight the impact that obesity has on the hepatic function in pregnant women by comparing the functional tests used in current practice. In addition, the aim was to identify possible predictors of liver damage by analyzing specific anthropometric data. The present study was descriptive, observational, retrospective, and based on the observation sheets found in the database of the Institute for the Health of the Mother and Child, the Obstetrics Gynecology Department of Polizu Hospital. Patients who presented for consultation in each trimester of pregnancy were included in the study. Demographic data taken into account included age, body mass index (BMI), provenance environment, anthropometric data: Abdominal circumference and the complete set of paraclinical data from which we extracted these specific liver tests: Aspartate aminotransferase (AST), alanine transferase (ALT), direct bilirubin (BD), serum albumin and gamma-glutamyl transferase (GGT). The present study included 157 patients divided into two groups, distributed as follows: Group A: 66 obese pregnant women $\left(\mathrm{BMI}>25 \mathrm{~kg} / \mathrm{m}^{2}\right)$ and group B: 91 patients with normal weight $\left(\mathrm{BMI}<25 \mathrm{~kg} / \mathrm{m}^{2}\right)$. Measurement of serum ALT and AST were the most useful tests for routine diagnosis of liver disease. The effects of pregnancy on serum levels of ALT and AST are controversial. In some
\end{abstract}

Correspondence to: Dr Florica Şandru, Department of Dermatology, 'Elias' Emergency University Hospital, Bd. Mărăști 17, 011461 Bucharest, Romania

E-mail: florysandru@yahoo.com

Key words: pregnancy, obesity, liver functional tests, body mass index, abdominal circumference studies, there was a slight increase in ALT and AST during the second and third trimesters, a fact confirmed by our study, albeit the result was not statistically significant Most published studies claim that serum ALT and AST levels do not change during pregnancy. In conclusion, obesity during pregnancy does not drastically influence liver function. However, patients with greater abdominal circumference are prone to developing minor hepatic cytolysis syndrome during the gestation period. The liver functional tests described in the aforementioned groups agree with the results provided by the specialized studies.

\section{Introduction}

The World Health Organisation (WHO) defined obesity as one of the most dangerous and yet neglected public health issues which threaten to overwhelm even the most developed countries (1). The association between obesity and a higher cancer risk is mainly due to anthropometric parameters and lifestyle factors which activate different biological mechanisms. Anthropometric parameters are BMI, weight increase, and the amount of body fat, particularly visceral fat. Lifestyle factors include sedentary habits and diet parameters, such as a hypercaloric and/or low-quality diet (2). Obesity during pregnancy is an independent risk factor for long-term female malignancies such as ovarian and breast cancer (3). As with obesity, cancer has been acknowledged worldwide as a leading disease with respect to the total number of fatalities, which are mostly caused by late diagnosis (4). Regarding breast cancer prevention, one important aspect lies in the use of screening methods such as mammography. Concerning this, the major issue is related to the filter used in mammographies in that the filters must have homogen mass densities, to ensure the uniform exposure of the patient's breast (5). Cancer is characterized by uncontrolled cell division. Numerous solutions to this include the use of cytostatic agents and the development of theoretical models that describe the use 
Table I. Comparative analysis of the AST and ALT levels in the two groups according to the pregnancy trimester.

\begin{tabular}{lcccccc}
\hline Paraclinical data & Mean group A & Mean group B & 95\% CI group A & $\begin{array}{c}\text { 95\% CI } \\
\text { group B }\end{array}$ & $\begin{array}{c}\text { Standard error } \\
\text { of difference }\end{array}$ & P-value \\
\hline AST T1 & 33.2459 & 25.2623 & 29.2985 to 37.1933 & 21.6698 to 28.8548 & 1.97 & 0.1 \\
AST T2 & 34.3115 & 32.8197 & 30.8527 to 37.7703 & 29.1720 to 36.4674 & 2.757 & 1.0 \\
AST T3 & 34.0656 & 29.2459 & 30.4825 to 37.6487 & 26.9635 to 31.5283 & 2.135 & 0.4 \\
ALT T1 & 27.1270 & 28.0476 & 23.8323 to 30.4217 & 24.3658 to 31.7294 & 2.569 & 1.0 \\
ALT T2 & 32.8889 & 32.8730 & 29.2861 to 36.4917 & 29.3522 to 36.3938 & 2.775 & 1.0 \\
ALT T3 & 31.8413 & 32.5238 & 28.5619 to 35.1206 & 29.4757 to 35.5719 & 2.359 & 1.0 \\
\hline
\end{tabular}

AST, aspartate transferase; ALT, alanine aminotransferase; T1, first; T2, second; T3, third trimester of pregnancy.

of electrostatic fields in in this regard (6,7). Therefore, it is imperative to put effort into both cancer treatment as well as cancer prevention. Of importance is that there are no approved clinical guidelines for managing obesity during pregnancy $(8,9)$.

The main objective of this study was to emphasize the impact of obesity on hepatic function in pregnant women by analysing the functional tests used in current practice. A further aim was to identify possible predictors of liver damage by analyzing specific anthropometric data (10). There was also a focus on visceral fat which is associated with a number of conditions, including cardiovascular disease, and insulin resistance during the process of visceral adipose tissue collection in the abdominal cavity and surrounding of the internal organs (11).

\section{Materials and methods}

General. This study is descriptive, observational and retrospective and is based on the observation sheets found in the database of the Institute for the Health of the Mother and Child, the Obstetrics Gynecology Department of Polizu Hospital. Patients who presented for consult each trimester of pregnancy were included. The general demographic data considered included age, body mass index (BMI), area of origin, and anthropometric data: Abdominal circumference and a complete set of paraclinical data from which we extracted these specific liver tests: Aspartate aminotransferase (AST), alanine transferase (ALT), direct bilirubin (BD), serum albumin and gamma-glutamyl transferase (GGT) (6-9). In order to reduce the possibility of bias and to increase the reproducibility of the study the normal values of these parameters were produced: Direct bilirubin $<1.2 \mathrm{ml} / \mathrm{dl}$, AST $<35 \mu / 1$, ALT $<35 \mu / 1$, albumin 3.5-4.5 $\mu / 1$, GGT <36 $\mu / 1$ (12-18). It should be considered that AST and ALT levels were monitored dynamically, over several days, describing the variation pattern of the AST value and respecting the cubic model with a significance value of $\mathrm{P}=0.01$. This study was approved by the ethics commission of the National Institute for Maternal and Child Health-Alessandrescu Rusescu on December 5, 2019 with reference number 17852/07.11.2019.

Methods. Given the main end-point of this study, which is the comparison of liver function tests during pregnancy in patients with normal weight and overweight/obese, the size of the patient groups should be sufficient to meet this aim $(19,20)$. For this estimation the program MedCalc 14.1 (sampling-comparison of two means test) was used. A significance level of 0.05 was employed to avoid a type 1 ( $\alpha$ level-two sided) error and 0.1 to avoid a type $2(\beta)$ error. Thus, 157 patients (66 in group A and 91 in group B) were included in order to guarantee the detection of a $30 \%$ difference between the averages of the two groups with a computing power equal to $90 \%$. Written informed consent was obtained from all the patients.

Statistical analysis. For the statistical analysis of the data, the program MedCalc 14.1 (Stata Corp.) was used as follows: The t-test for independent variables to compare the differences between two means, one-way-repeated measures ANOVA for comparing the differences between three or more means using the Bonferroni correction as post hoc test, and the Pearson or Spearman correlation coefficient depending on observing the Gaussian distribution of data. For all analysis, the confidence interval was $95 \%$; thus, $\mathrm{P}<0.05$ was considered to indicate statistical significance.

\section{Results}

General. The present study included 157 patients divided into two groups, distributed as follows: Group A: 66 obese pregnant women (BMI $\left.>25 \mathrm{~kg} / \mathrm{m}^{2}\right)$ and group B: 91 patients with normal weight (BMI $<25 \mathrm{~kg} / \mathrm{m}^{2}$ ).

AST and ALT levels. Comparative analysis of the AST level in the two groups according to the trimester of pregnancy. When comparing the AST level, it can be observed that obese patients (group A) tend to have higher values in all trimesters, but no difference compared with group B was statistically significant (only in the first trimester is a slight difference detectable between the two groups $\mathrm{P}=0.1$ ) (Table I and Fig. 1). Comparative analysis of the ALT level in the two groups according to the trimester of pregnancy revealed no statistically significant differences in the comparative analysis between the two groups of ALT (Fig. 2).

$G G T$, albumin and BD levels. Comparative analysis of the GGT level in the two groups was conducted according to the pregnancy trimester. The values of the GGT are higher in 


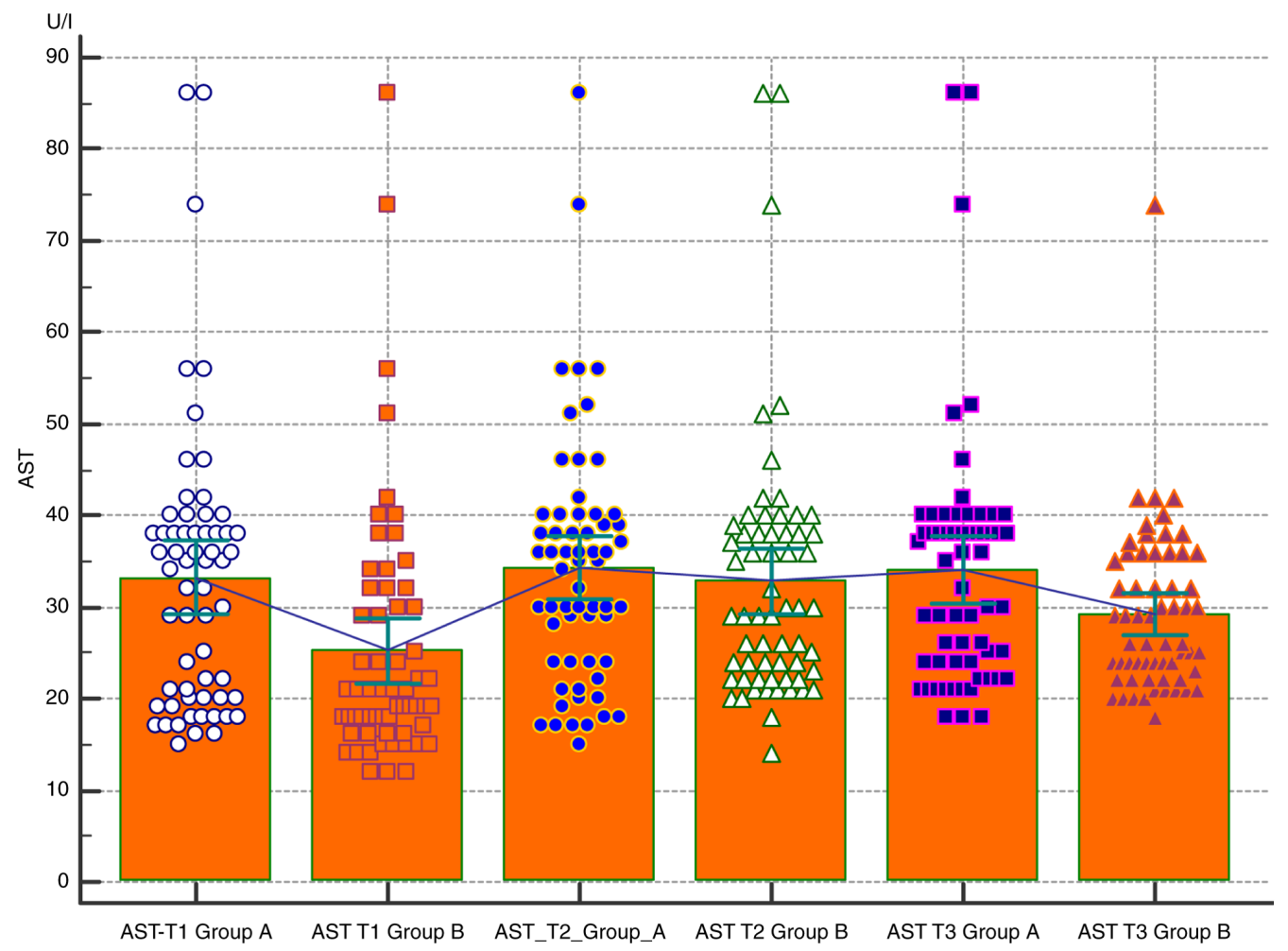

Figure 1. Comparative analysis of the AST level in the two groups according to the trimester of pregnancy.

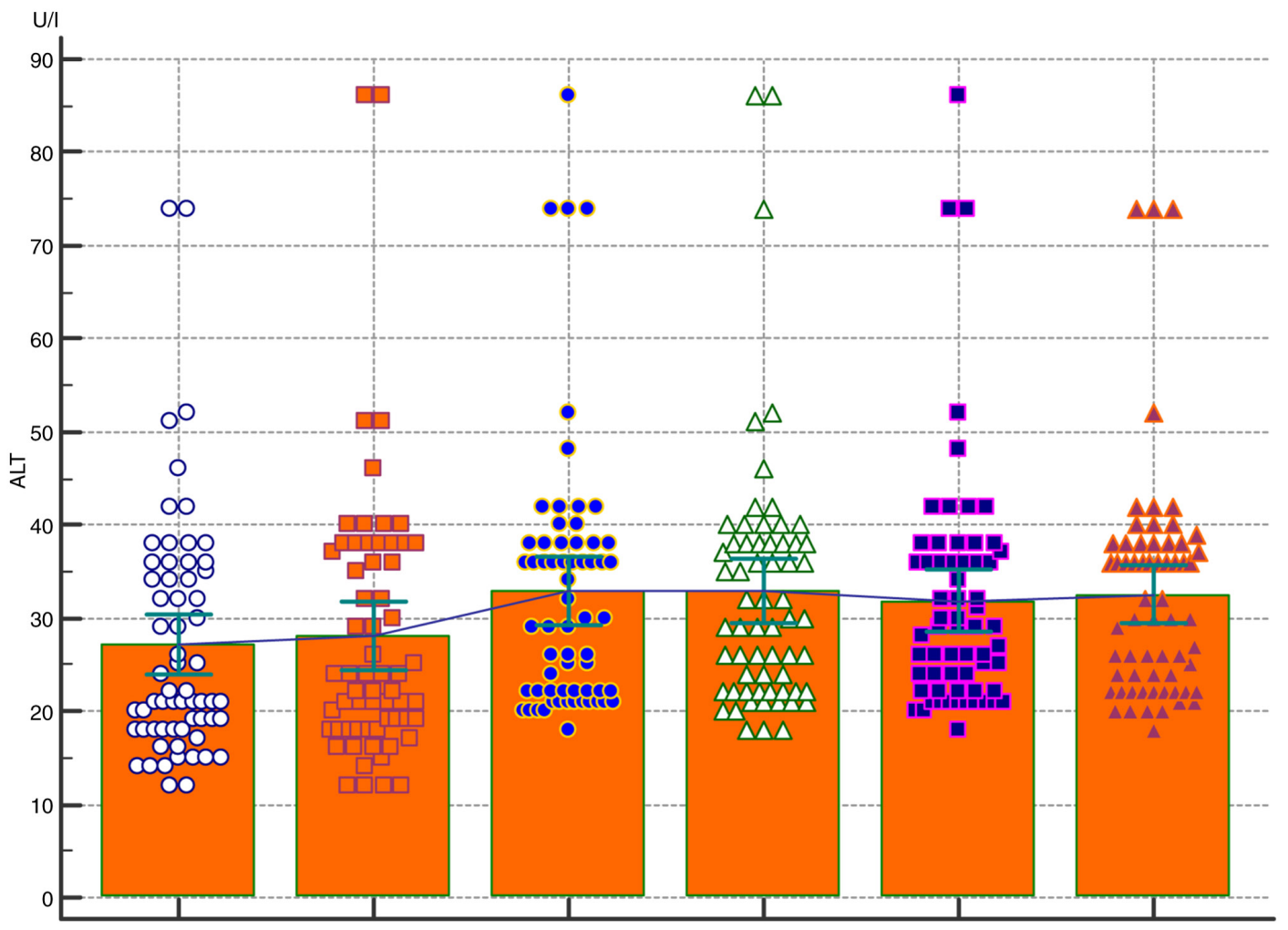

ALT_T1_Group_A ALT_T1_Group_B ALT_T2_Group_A ALT_T2_Group_B ALT_T3_Group_A ALT_T3_Group_B

Figure 2. Comparative analysis of the ALT level in the two groups according to the trimester of pregnancy.

the group of obese patients compared to the normoponderous patients, this difference being best underlined in the third trimester (Table II and Fig. 3).
Comparative analysis of the albumin level in the two groups according to the pregnancy trimester was also performed (Fig. 4). The level of albumin remains constant at the lower 


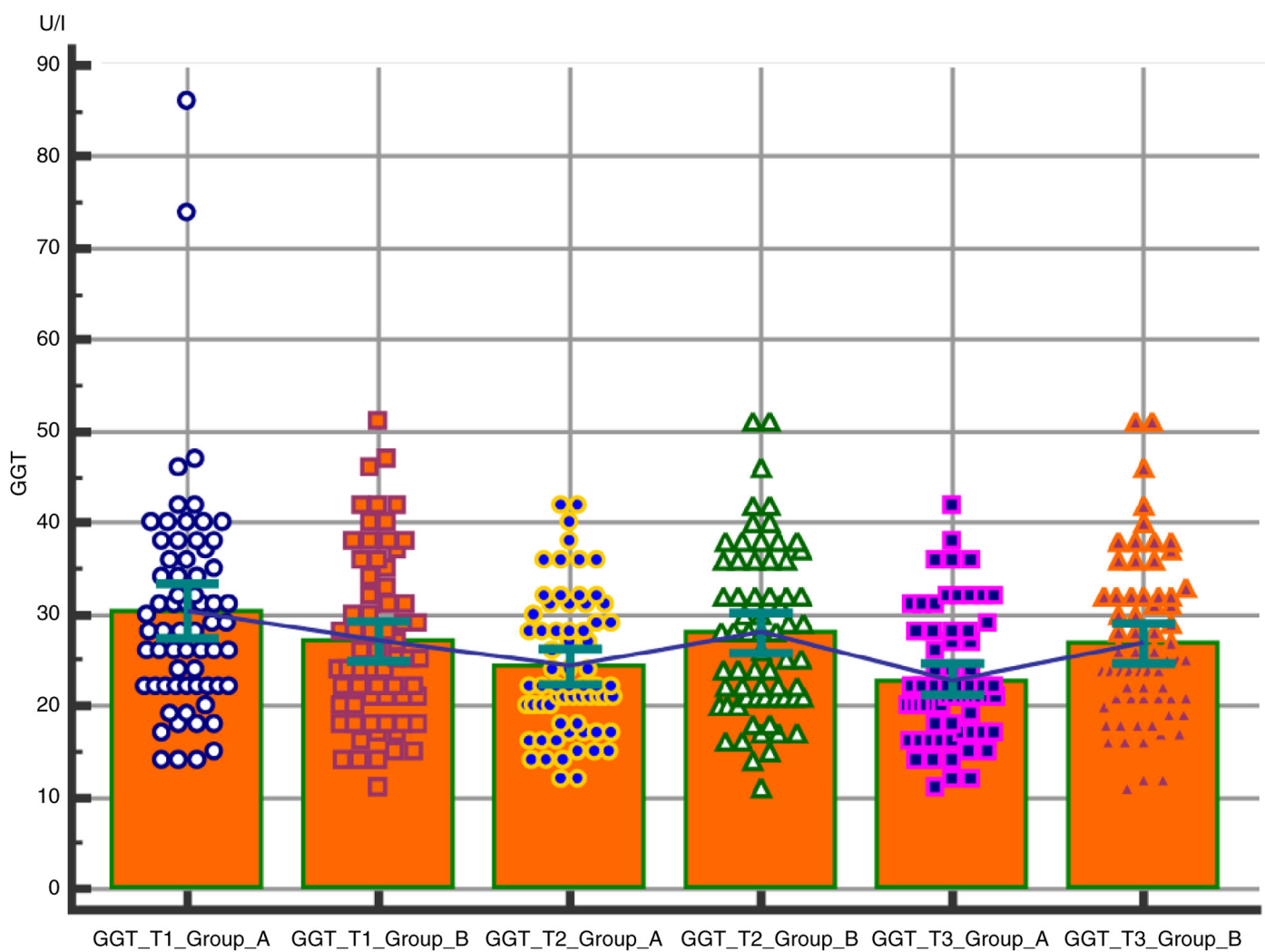

Figure 3. Comparative analysis of the GGT level in the two groups according to the pregnancy trimester.

Table II. Pelvic Floor Distress Inventory-20 and Pelvic Floor Impact Questionnaire-scores in obese and non-obese patients.

\begin{tabular}{lccc}
\hline Variable & Non-obese $(\mathrm{n}=44)$ & Obese $(\mathrm{n}=30)$ & $\mathrm{P}$-value \\
\hline PFIQ-7 & Mean=152.72 & Mean=191.73 & $\mathrm{P}=0.0034$ \\
& $\mathrm{SD}=69.16$ & $\mathrm{SD}=60.00$ & $\mathrm{t}=3.0248$ \\
& & & $\mathrm{df}=72$ \\
PFDI-20 & Mean=156.84 & Mean=192.5 & $\mathrm{P}=0.0097$ \\
& $\mathrm{SD}=64.33$ & $\mathrm{SD}=43.12$ & $\mathrm{t}=2.6535$ \\
& & & $\mathrm{df}=72$ \\
Prolapse degree & $\mathrm{I}-\mathrm{II}=25$ & $\mathrm{I}-\mathrm{II}=8$ & \\
& $\mathrm{III}-\mathrm{IV}=19$ & $\mathrm{III}-\mathrm{IV}=22$ & \\
\hline
\end{tabular}

PFIQ, Pelvic Floor Impact Questionnaire (scale 0-300); PFDI, Pelvic Floor Distress Inventory (scale 0-300); SD, standard deviation.

limit of the normal value of $3.5 \mu / 1$ regardless of the nutritional status. There is no statistically significant difference between the two groups.

Comparative analysis of the BD level in the two groups according to the pregnancy trimester was carried out. As in the case of albumin, direct bilirubin tends to be constant throughout pregnancy, with no statistically significant difference between the group of obese patients and the group of norm-weight patients (Fig. 5 and Table II).

Correlation of circumference and AST. The correlation between the abdominal circumference in the first trimester
Table III. Correlation between abdominal circumference in the first trimester and the value of aspartate transferase.
Sample size

Correlation coefficient $r$

Significance level

95\% confidence interval for $\mathrm{r}$
157

0.1997

$\mathrm{P}=0.0122$

0.04444 to 0.3455 and the value of aspartate transferase was assessed. In practice, in a patient with a larger abdominal circumference in the first trimester, higher levels of AST were evident (Table III).

\section{Discussion}

ALT and AST. Measurement of serum ALT and AST were the most useful tests for routine diagnosis of liver disease. The effects of pregnancy on serum levels of ALT and AST are considered somewhat controversial. In some studies, there was a slight increase in ALT and/or AST during the second and third trimesters. However, in most published studies, serum ALT and AST levels did not change during pregnancy $(21,22)$.

Results of the present study showed that obese pregnant women tend to have higher values of hepatic transaminases during all 3 trimesters of pregnancy compared to normal weight pregnant women, even though it was not statistically significant. However, it should be considered that AST and ALT levels were monitored dynamically, over several days; thus, it is necessary to describe the variation pattern of 


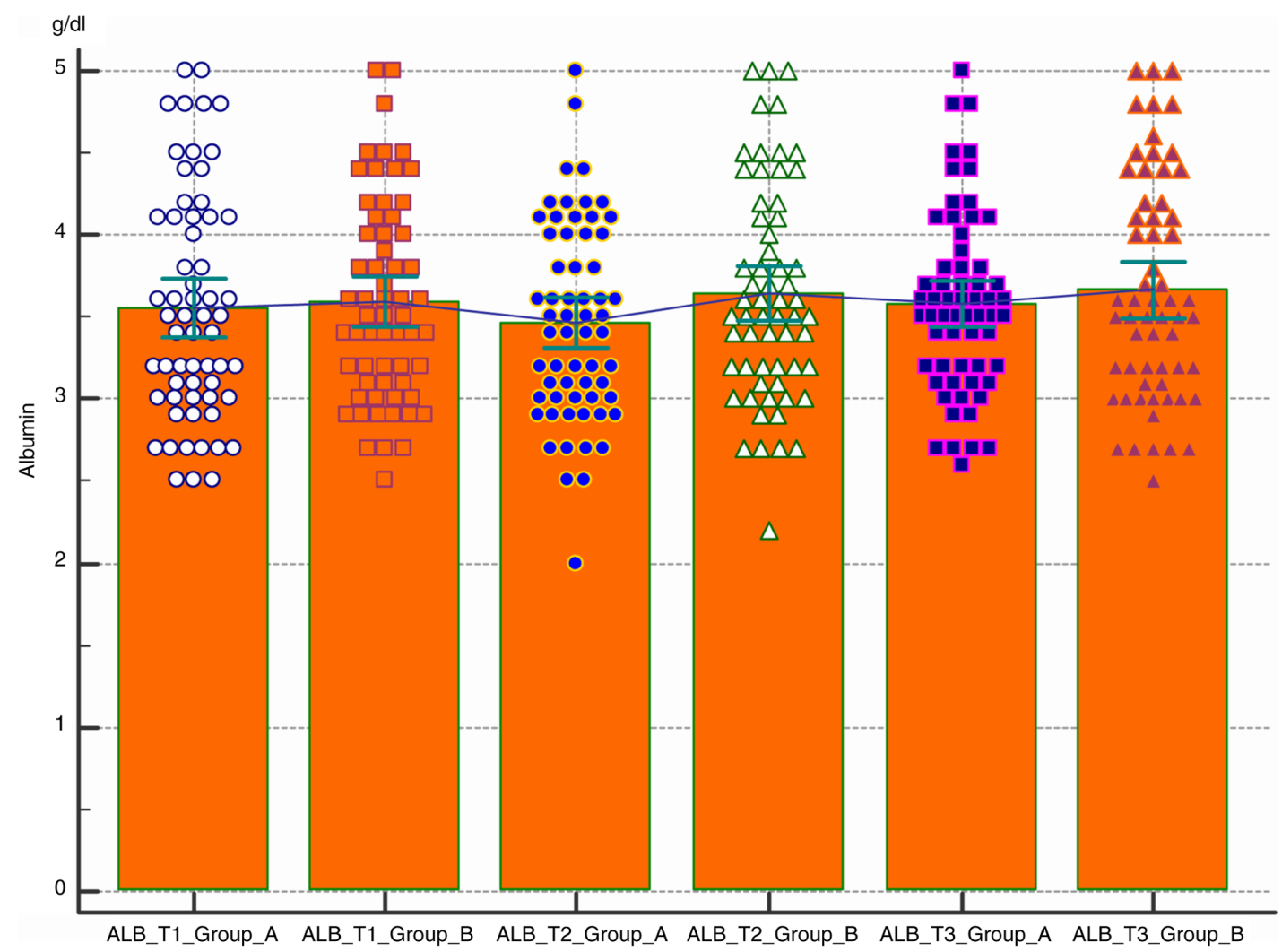

Figure 4. Comparative analysis of the albumin level in the two groups according to the pregnancy trimester.

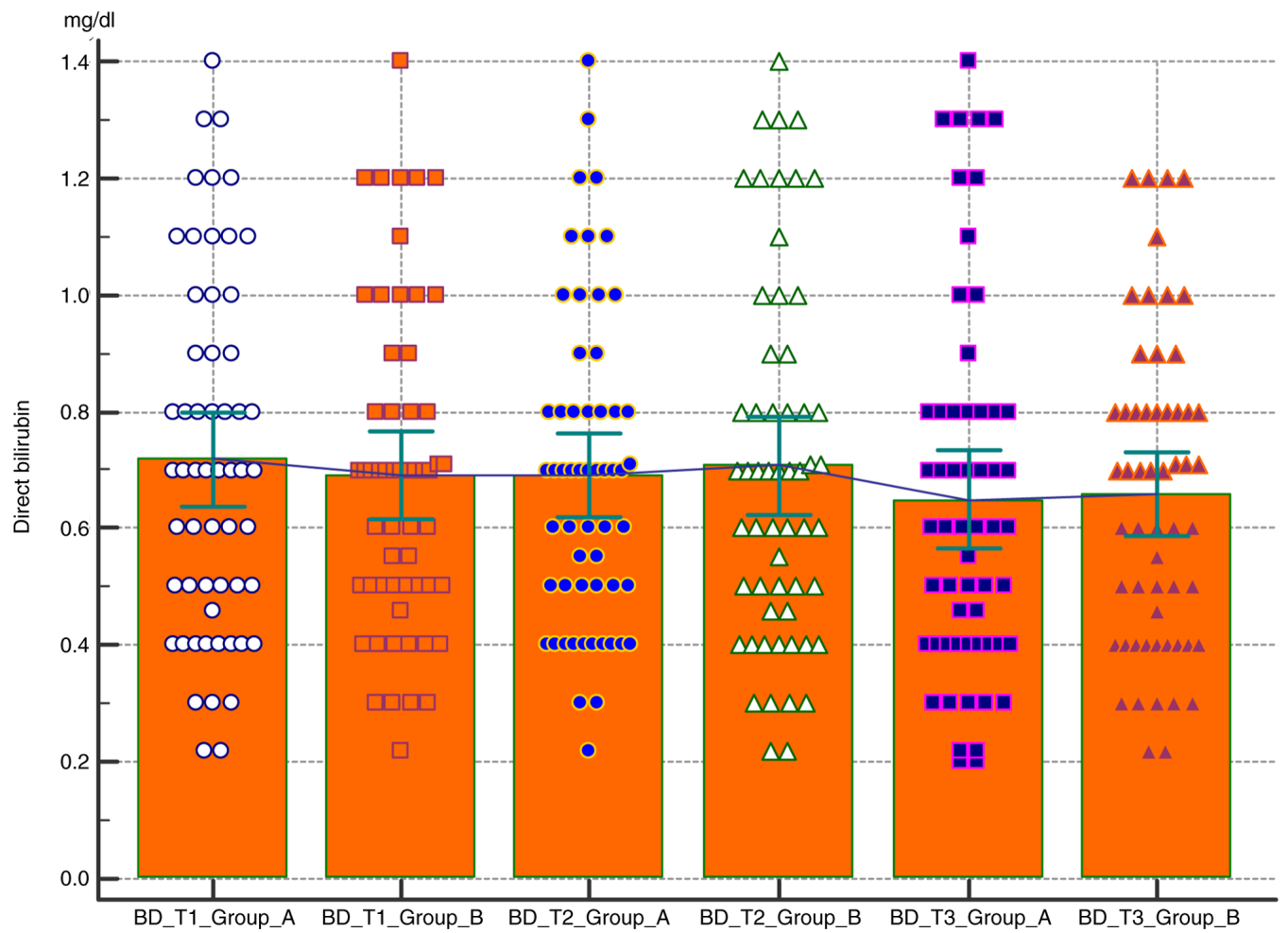

Figure 5. Comparative analysis of the $\mathrm{BD}$ level in the two groups according to the pregnancy trimester.

the AST value (23-29). It respects the cubic model with a significance value of $\mathrm{P}=0.01$, which shows that during our follow-up, the AST values tended to increase during Q2, followed by a definite decrease. However, the AST values in Q3 are higher than those in Q1 (Table I and Fig. 1). In addition, there were no significant statistical differences in the 


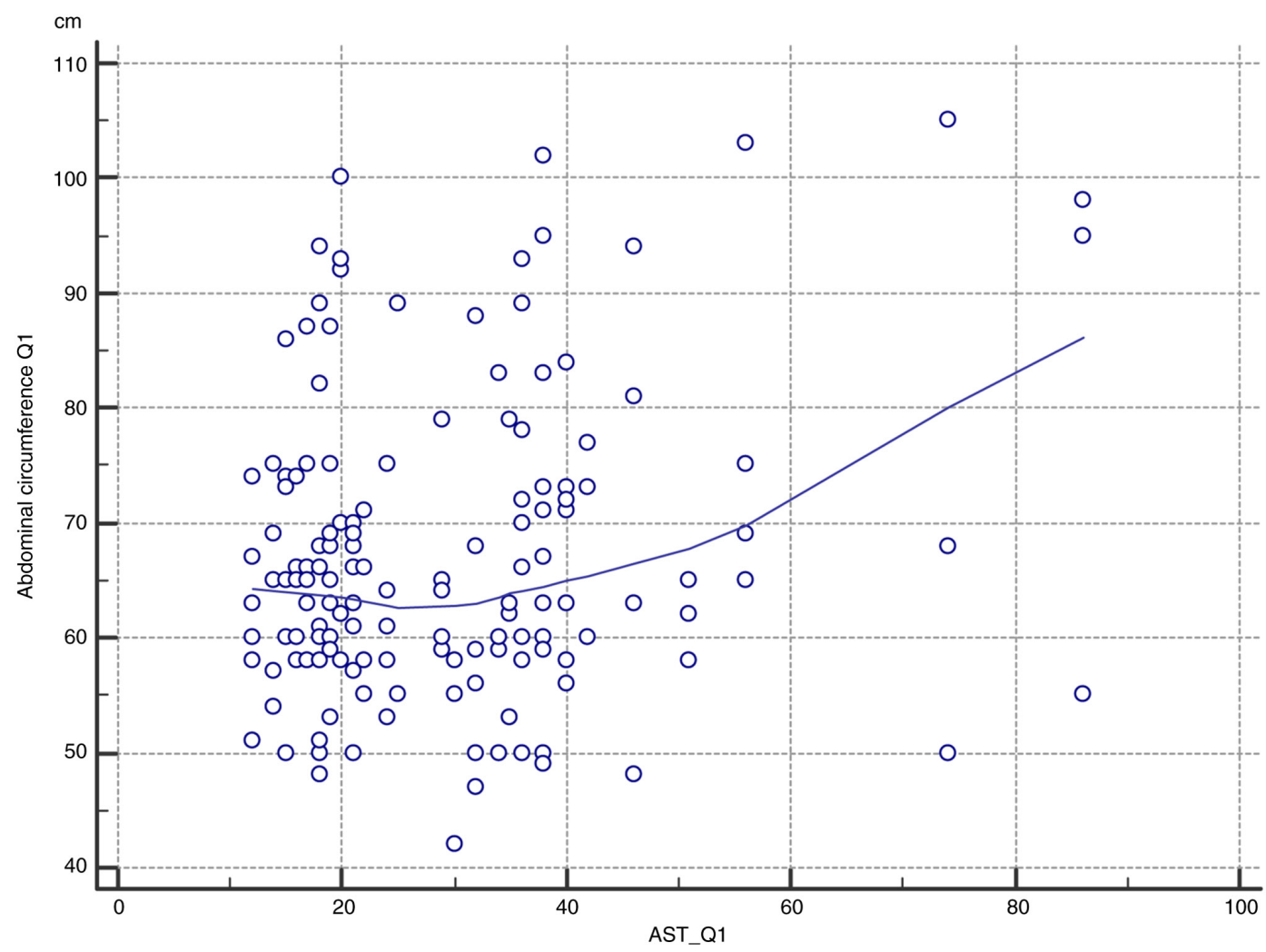

Figure 6. Correlation between abdominal circumference in the first trimester and the value of aspartate transferase.

comparative analysis between the two groups regarding AST values. The evolution trend was linear with a significance level of $\mathrm{P}=0.05$ (Fig. 2).

The GGT values of were higher in the group of obese patients (group A) compared to the normoponderous patients, this difference being particularly highlighted in the third trimester (Table II and Fig. 3).

Both albumin and direct bilirubin levels have relative constant values throughout pregnancy, with albumin levels remaining close to the lower normal limits $(3.5 \mu / 1)$. However, none of these values indicated statistical significance.

Given the fact that the only liver function test that varies between the two groups is AST, we performed a Pearson correlation analysis; the data studied concerns the Gaussian distribution. Thus, a directly proportional result and a significant Pearson correlation $(\mathrm{r}=0.19 ; \mathrm{P}=0.012)$ were obtained. Furthermore, a larger abdominal circumference is associated with higher levels of AST during T1 pregnancy (Table III and Fig. 6).

In conclusion, obesity during pregnancy does not markedly influence the patient's liver function. However, patients with greater abdominal circumference are prone to developing a slight hepatic cytolysis syndrome during pregnancy. The functional tests of the liver that we used in the present study agree with the results provided by the specialized studies.

\section{Acknowledgements}

This study, together with the efforts in article publishing were self-founded.

\section{Funding}

No funding was received.

\section{Availability of data and materials}

The datasets used and analyzed during the current study are available from the corresponding author on reasonable request.

\section{Authors' contributions}

CODT analyzed and interpreted the patients' data regarding the hepatic function by comparing the functional tests used in current practice and critically revised the manuscript for its content.FS analyzed and interpreted the patients' data regarding the hepatic function by comparing the functional tests used in current practice and is the corresponding author. AC identified the possible predictors of liver damage and wrote the manuscript. AT revised the literature data and analyzed specific anthropometric patient data. ARP researched the papers that were included as references. AIM designed the experiments and critically revised the manuscript and approved the current form of the article in order to be submitted to the journal. All authors read and approved the final manuscript.

\section{Ethics approval and consent to participate}

This study was approved by the ethics commission of the National Institute for Maternal and Child Health 'Alessandrescu Rusescu' on December 5th, 2019 with ref. 
no. 17852/07.11.2019. Written informed consent was provided by the patients.

\section{Patient consent for publication}

The patients have given written informed consent for the publication of the data.

\section{Competing interests}

The authors declare that they have no competing interests.

\section{References}

1. WHO. Geneva, Switzerland: World Health Organization, 2006. https://www.who.int/whosis/whostat2006.pdf.

2. De Pergola G and Silvestris F: Obesity as a major risk factor for cancer. J Obes 2013: 291546, 2013.

3. Kessous R, Davidson E, Meirovitz M, Sergienko R and Sheiner E: Prepregnancy obesity: A risk factor for future development of ovarian and breast cancer. Eur J Cancer Prev 26: 151-155, 2017.

4. Verga N, Mirea DA, Busca I, Poroschianu MN, Zarma SF, Grinisteanu L, Anica A, Gheorghe CA, Stan CA, Verga M and Vasilache R: Optical coherence tomography in oncological imaging. Rom Rep Phys 66: 75-86, 2014.

5. Scarlat F, Scarisoreanu A and Verga N: Absorbed dose distributions using the isodensitometric method for exposures with filter employed for mammographies. Rom Rep Phys 65: 168-177, 2013.

6. Sardari D and Verga N: Calculation of externally applied electric field intensity for disruption of cancer cell proliferation. Electromagn Biol Med 29: 26-30, 2010.

7. Sardari D and Verga N: A physical model for study of electromagnetic field interaction with cancer cell. Prog Electromagn Res Symp 2: 891-893, 2008.

8. Adams EJ, Grummer-Strawn L and Chavez G: Food insecurity is associated with increased risk of obesity in California women. J Nutr 133: 1070-1074, 2003.

9. Rooney BL and Schauberger CW: Excess pregnancy weight gain and long-term obesity: One decade later. Obstet Gynecol 100: 245-252, 2002 .

10. Lynch CM, Sexton DJ, Hession M and Morrison JJ: Obesity and mode of delivery in primigravid and multigravid women. Am J Perinatol 25: 163-167, 2008.

11. Dumitrascu MC, Stanescu AMA, Bejan C, Sandru F, Toader DO, Radavoi DG, Cotirlet A, Judea-Pusta CT and Diaconu CC: Obesity and its Implications on Stress Urinary Incontinence. Rev Chim 70: 3660-3662, 2019.

12. O'Brien TE, Ray JG and Chan WS: Maternal body mass index and the risk of preeclampsia: A systematic overview. Epidemiology 14: 368-374, 2003.
13. Soens MA, Birnbach DJ, Ranasinghe JS and van Zundert A: Obstetric anesthesia for the obese and morbidly obese patient: An ounce of prevention is worth more than a pound of treatment. Acta Anaesthesiol Scand 52: 6-19, 2008.

14. Ehrenberg HM, Mercer BM and Catalano PM: The influence of obesity and diabetes on the prevalence of macrosomia. Am J Obstet Gynecol 191: 964-968, 2004.

15. Hedderson MM,Williams MA, Holt VL, Weiss NS and Ferrara A: Body mass index and weight gain prior to pregnancy and risk of gestational diabetes mellitus. Am J Obstet Gynecol 198: 409. e1-e7, 2008.

16. Chu SY, Callaghan WM, Kim SY, Schmid CH, Lau J, England LJ and Dietz PM: Maternal obesity and risk of gestational diabetes mellitus. Diabetes Care 30: 2070-2076, 2007.

17. Radaelli T, Varastehpour A, Catalano P and Hauguel-de Mouzon S: Gestational diabetes induces placental genes for chronic stress and inflammatory pathways. Diabetes 52 : 2951-2958, 2003

18. Yogev Y and Langer O: Pregnancy outcome in obese and morbidly obese gestational diabetic women. Eur J Obstet Gynecol Reprod Biol 137: 21-26, 2008.

19. Usha Kiran TS, Hemmadi S, Bethel J and Evans J: Outcome of pregnancy in a woman with an increased body mass index. BJOG 112: 768-772, 2005.

20. Brown CD, Higgins M, Donato KA, Rohde FC, Garrison R, Obarzanek E, Ernst ND and Horan M: Body mass index and the prevalence of hypertension and dyslipidemia. Obes Res 8: $605-619,2000$

21. de Ferranti S and Mozaffarian D: The perfect storm: Obesity, adipocyte dysfunction, and metabolic consequences. Clin Chem 54: 945-955, 2008.

22. Boden G: Fatty acid-induced inflammation and insulin resistance in skeletal muscle and liver. Curr Diab Rep 6: 177-181, 2006.

23. Boden G: Role of fatty acids in the pathogenesis of insulin resistance and NIDDM. Diabetes 46: 3-10, 1997.

24. Hotamisligil GS and Erbay E: Nutrient sensing and inflammation in metabolic diseases. Nat Rev Immunol 8: 923-934, 2008.

25. Lederman SA, Paxton A, Heymsfield SB, Wang J, Thornton J and Pierson RN Jr: Body fat and water changes during pregnancy in women with different body weight and weight gain. Obstet Gynecol 90: 483-488, 1997.

26. Okereke NC, Huston-Presley L, Amini SB, Kalhan S and Catalano PM: Longitudinal changes in energy expenditure and body composition in obese women with normal and impaired glucose tolerance. Am J Physiol Endocrinol Metab 287: E472-E479, 2004.

27. Kopp-Hoolihan LE, van Loan MD, Wong WW and King JC: Fat mass deposition during pregnancy using a four-component model. J Appl Physiol (1985) 87: 196-202, 1999.

28. Dumitrascu MC, Iliescu M, Petca RC, Sandru F, Mehedintu P, Farcasanu PD, Maru N, Chibelean C and Petca A: The chemical pregnancy. Rev Chim 70: 3818-3823, 2019.

29. Alese MO, Moodley J and Naicker T: Preeclampsia and HELLP syndrome, the role of the liver. J Matern Fetal Neonatal Med 34: 117-123, 2021. 